OPEN ACCESS

Edited by:

Mariusz Cycoń,

Medical University of Silesia, Poland

Reviewed by:

Qian Guoliang,

Nanjing Agricultural University, China

Qing Yan,

Oregon State University,

United States

Wenli Chen,

Huazhong Agricultural University,

China

${ }^{*}$ Correspondence:

Hai-Lei Wei

weihailei@caas.cn;

hw359@cornell.edu

Specialty section:

This article was submitted to Microbiotechnology, Ecotoxicology

and Bioremediation,

a section of the journal

Frontiers in Microbiology

Received: 13 September 2018

Accepted: 23 November 2018

Published: 10 December 2018

Citation:

Xia Z, Lei L, Zhang $\mathrm{H}-\mathrm{Y}$ and

Wei H-L (2018) Characterization of the ModABC Molybdate Transport

System of Pseudomonas putida

in Nicotine Degradation.

Front. Microbiol. 9:3030

doi: 10.3389/fmich.2018.03030

\section{Characterization of the ModABC Molybdate Transport System of Pseudomonas putida in Nicotine Degradation}

\author{
Zhenyuan Xia ${ }^{1,2}$, Liping Lei ${ }^{2}$, Hong-Yue Zhang ${ }^{1}$ and Hai-Lei Weit* \\ ${ }^{1}$ Key Laboratory of Microbial Resources Collection and Preservation, Ministry of Agriculture, Institute of Agricultural \\ Resources and Regional Planning, Chinese Academy of Agricultural Sciences, Beijing, China, ${ }^{2}$ Yunnan Academy of Tobacco \\ Agricultural Science, Kunming, China
}

Pseudomonas putida $\mathrm{J} 5$ is an efficient nicotine-degrading bacterial strain that catabolizes nicotine through the pyrrolidine pathway. In our previous study, we used Tn5 transposon mutagenesis to investigate nicotine metabolism-associated genes, and 18 nicotine degradation-deficient mutants were isolated from 16,324 Tn5-transformants. Three of the mutants were Tn5 inserts into the modABC gene cluster that encoded an $A B C$-type, high-affinity, molybdate transporter. In-frame deletion of the modABC genes abolished the nicotine-degrading ability of strain $\mathrm{J} 5$, and complementation with modABC either from $P$. putida or Arthrobacter oxidans restored the degrading activity of the mutant to wild-type level. Nicotine degradation of J5 was inhibited markedly by addition of tungstate, a specific antagonist of molybdate. Molybdate at a nonphysiologically high concentration $(100 \mu \mathrm{M})$ fully restored nicotine-degrading activity and recovered growth of the modABC mutant in a nicotine minimal medium. Transcriptional analysis revealed that the expression of $\bmod A B C$ was up-regulated at low molybdate concentrations and down-regulated at high moybdate concentrations, which indicated that at least one other system was able to transport molybdate, but with lower affinity. These results suggested that the molybdate transport system was essential to nicotine metabolism in P. putida J5.

Keywords: biodegradation, nicotine, Pseudomonas putida, molybdate transporter, ModABC

\section{INTRODUCTION}

Molybdenum (Mo) plays essential roles in bacteria, because it serves as a cofactor for a number of enzymes that catalyze a variety of oxidation/reduction reactions, and it is involved in microbial metabolism of carbon, nitrogen, and sulfur (Hille, 1996; Kisker et al., 1997). For the synthesis of molybdoenzymes, bacteria need to transport molybdate, activate it to an appropriate form, and incorporate it into the organic part of the molybdenum cofactor (Zhang et al., 2011). In nature, 
the predominant form of Mo is molybdate oxyanion, which is transported by an ABC-type transport system (Self et al., 2001). In Escherichia coli, the first identified Mo transporter was the high affinity ModABC transport system, in which ModA was responsible for molybdate binding, ModB was the transmembrane component of the permease, and ModC provided the energizer function on the cytoplasmic side of the membrane (Maupin-Furlow et al., 1995). Based on genome sequences, mod homologs can be identified in $\geq 50$ bacteria (Leimkuhler and Iobbi-Nivol, 2015). Among these bacteria, mod genes have been functionally characterized in only a few species, which include Rhodobacter capsulatus (Wang et al., 1993), Azotobacter vinelandii (Luque et al., 1993), Staphylococcus carnosus (Neubauer et al., 1999), and Bradyrhizobium japonicum (Delgado et al., 2006). In B. japonicum USDA110, a nitrogenfixing, root-nodule symbiont of soybean, $\bmod A$ and $\bmod B$ mutant strains were unable to grow with nitrate as a nitrogen source or as a respiratory substrate, and they lacked nitrate reductase activity (Delgado et al., 2006). Partially purified quinoline dehydrogenase from $P$. putida Chin IK indicated the presence of favin and molybdenum-binding pterin, and the enzyme activity was wholly dependent on the availability of molybdate in the growth medium (Blaschke et al., 1991).

Mo uptake occurred during nicotine biodegradation by grampositive bacteria (Freudenberg et al., 1988; Menendez et al., 1997). The nicotine utilization of Arthrobacter oxidans P-34 (DSM419) required a supplementation with molybdate to the growth medium, and nicotine dehydrogenase (NDH) was identified as a molybdo-iron-sulfur-flavoprotein (Freudenberg et al., 1988). The nicotine-degrading plasmid pAO1 of $A$. nicotinovorans was sequenced and showed a remarkable gene cluster for biosynthesis of the molybdenum cytosine dinucleotide (MCD) cofactor and molybdate transporter system that were involved in nicotine degradation (Ganas et al., 2008). Despite the importance of Mo in nicotine metabolism by gram-positive bacteria, there has been very little work on the mechanisms involved in uptake of molybdate in nicotine metabolism by gram-negative bacteria. A recent report showed that conversion of 3-succinoylpyridine to 6-hydroxy-3-succinoylpyridine during the degradation of nicotine by $P$. putida $\mathrm{S} 16$ was catalyzed by a multi-enzyme reaction that consisted of a molybdopeterin binding oxidase (SpmA), molybdopterin dehydrogenase (SpmB), and a (2Fe-2S)-binding ferredoxin (SpmC) with molybdenum molybdopterin cytosine dinucleotide as a cofactor (Tang et al., 2013). This result led us to presume that Mo uptake was also involved in nicotine metabolism by gram-negative bacteria.

Pseudomonas putida J5 is an efficient nicotine-degrading strain isolated from the tobacco rhizosphere which could catabolize $3 \mathrm{~g} / \mathrm{l}$ nicotine in $24 \mathrm{~h}$ (Wei et al., 2008). In our most recent study on nicotine-degrading associated genes in $P$. putida J5, we generated a Tn5 transposon mutant library with 16,324 transformants and screened 18 nicotinedegrading deficient mutants in which three mutants were mutated directly by $\operatorname{Tn} 5$ in the $\bmod A B C$ transport gene cluster (Xia et al., 2015). In this paper, we cloned the entire gene cluster that encodes the molybdate transport system from strain J5, in-frame deleted the $\bmod A B C$ genes, and characterized the function of the ModABC system in nicotine metabolism.

\section{MATERIALS AND METHODS}

\section{Strains, Plasmids, and Growth Conditions}

Characteristics of strains and plasmids are listed in Table 1. Pseudomonas putida was grown in Luria-Bertani (LB) medium or NI medium at $28^{\circ} \mathrm{C}$ (Wei et al., 2009; Xia et al., 2015), and Escherichia coli was grown at $37^{\circ} \mathrm{C}$ in $\mathrm{LB}$ medium. For plasmid propagation and selection of transformants, media were supplemented with antibiotics at appropriate concentrations as follows: $100 \mu \mathrm{g} / \mathrm{ml}$ ampicillin, $50 \mu \mathrm{g} / \mathrm{ml}$ kanamycin, and $20 \mu \mathrm{g} / \mathrm{ml}$ tetracycline.

\section{DNA Manipulations and Sequencing}

Chromosomal and plasmid DNA isolations, restriction enzyme digestions, agarose gel electrophoresis, ligations, and E. coli transformations were performed according to standard protocols (Sambrook and Russell, 1998). Nucleotide sequencing was performed by Invitrogen Co., Ltd., China.

\section{Southern Hybridization and Cloning of the Tn5 Insertion Sites}

To determine the copy number of the Tn5 transposon in mutant strains, total DNA was digested with EcoRI, PstI, and BamHI separated by electrophoresis on $0.8 \%$ $(\mathrm{w} / \mathrm{v})$ agarose gel and transferred onto nylon membranes (Hybond-N+; Amersham, GE Healthcare, Piscataway, NJ, United States). A digoxigenin-labeled, kanamycin-resistant, gene probe (Xia et al., 2015) was used to do hybridization and detection according to the protocol for the DIG High Primer DNA Labeling and Detection Starter Kit I (Roche).

Shotgun cloning was performed to determine the transposon insertion site. Chromosomal DNA samples were restricted with PstI and EcoRI and ligated into pBluescript II SK. E. coli $\mathrm{DH} 5 \alpha$ transformants were selected on LB medium that contained kanamycin. Positive clones were sequenced with Tn5-39 and Tn5-1571 (Xia et al., 2015) to allow determination of the precise location of a transposon insertion. Sequences were then compared to the protein sequence database (GenBank) using the BlastX algorithm (Altschul et al., 1990). For each mutant, the joins between the transposon sequences were identified.

\section{Construction of a modABC in-Frame Deletion Mutant}

To create a modABC gene deletion allele, two fragments that flanked $\bmod A B C$ genes were generated by endonuclease digestion. A $2.4 \mathrm{~kb}$ fragment was created by digestion with KpnI and SalI from pBS-M728, and another $1.6 \mathrm{~kb}$ 
TABLE 1| Bacterial strains and plasmids used in this study.

\begin{tabular}{|c|c|c|}
\hline Strain or plasmid & $\begin{array}{l}\text { Genotype or relative } \\
\text { phenotype }\end{array}$ & Source \\
\hline \multicolumn{3}{|l|}{ Escherichia coli } \\
\hline $\mathrm{DH} 5 \alpha$ & $\begin{array}{l}\text { F- recA1 endA1 hsdR17 } \\
\text { supE44 thi-1 gyrA96 } \\
\text { relA1 } \\
\text { (argF-lacZYA)l169Ф80lacZ } \\
\Delta \mathrm{M} 15\end{array}$ & $\begin{array}{l}\text { Sambrook and } \\
\text { Russell, } 1998\end{array}$ \\
\hline \multicolumn{3}{|l|}{ Pseudomonas putida } \\
\hline $\mathrm{J} 5$ & Apr; wild type & Wei et al., 2009 \\
\hline M430 & $\begin{array}{l}\text { Nicotine-degrading mutant } \\
\text { inserted with Tn5 }\end{array}$ & This study \\
\hline M728 & $\begin{array}{l}\text { Nicotine-degrading mutant } \\
\text { inserted with Tn5 }\end{array}$ & This study \\
\hline M9502 & $\begin{array}{l}\text { Nicotine-degrading mutant } \\
\text { inserted with Tn5 }\end{array}$ & This study \\
\hline$\triangle \bmod A B C$ & modABC deletion mutant & This study \\
\hline \multicolumn{3}{|l|}{ Arthrobacter oxidans } \\
\hline $\mathrm{J} 4$ & $\begin{array}{l}\text { Nicotine-degrading } \\
\text { bacterium }\end{array}$ & Lab collection \\
\hline \multicolumn{3}{|l|}{ Plasmids } \\
\hline pBluescript II SK+ & ColE1 origin; $A p^{r}$ & Stratagene \\
\hline pHSG299 & ColE1 origin; $\mathrm{Km}^{\mathrm{r}}$ & TaKaRa \\
\hline pRK2013 & $\begin{array}{l}\text { ColE1 replicon with RK2 } \\
\text { transfer region, helper } \\
\text { plamsid; } \mathrm{Km}^{r}\end{array}$ & $\begin{array}{l}\text { Figurski and } \\
\text { Helinski, } 1979\end{array}$ \\
\hline pRG970Km & $\begin{array}{l}\text { Cloning vector containing } \\
\text { promoterless lacZYA for } \\
\text { construction of } \\
\text { transcriptional fusions; } \mathrm{Km}^{\mathrm{r}}\end{array}$ & Yan et al., 2009 \\
\hline pME6032 & Shuttle vector; $T c^{r}$ & Heeb et al., 2000 \\
\hline pBBR1MCS-2 & Shuttle vector; $\mathrm{Km}^{\mathrm{r}}$ & Kovach et al., 199 \\
\hline pHSG299 $\Delta$ mod & pHSG299::modABC & This study \\
\hline p6032-modABC-J5 & $\begin{array}{l}\text { pME } 6032 \text { with full length of } \\
\text { modABC genes from J5 }\end{array}$ & This study \\
\hline p6032-modABC-J4 & $\begin{array}{l}\text { pME6032 with full length of } \\
\text { modABC genes from J4 }\end{array}$ & This study \\
\hline pMCS2-modABC-J5 & $\begin{array}{l}\text { pBBR1MCS- } 2 \text { with full } \\
\text { length of modABC genes } \\
\text { from J5 }\end{array}$ & This study \\
\hline pMCS2-modABC-J4 & $\begin{array}{l}\text { pBBR1MCS- } 2 \text { with full } \\
\text { length of modABC genes } \\
\text { from J4 }\end{array}$ & This study \\
\hline p970-PmodABC & $\begin{array}{l}\text { pRG970Km with a } 580 \mathrm{bp} \\
\text { fragment containing the } \\
\text { promoter of modABC } \\
\text { genes }\end{array}$ & This study \\
\hline
\end{tabular}

fragment was generated by digestion with XhoI and EcoRI from pBS-M9502. After being digested with relevant restriction enzymes, the two fragments were inserted into pBluescript to create pBS $\Delta$ mod. An approximately $4.0 \mathrm{~kb}$ long KpnI-EcoRI fragment, which included the modABC genes with a $1.8 \mathrm{~kb}$ deletion, was lifted and ligated into pHSG299. The last suicide plasmid pHSG299 $\Delta$ mod was introduced into $P$. putida J5 by electroporation, and this was used in a two-step strategy to introduce the shortened $\bmod A B C$ locus into the chromosome. Primers P3210
$\left(5^{\prime}\right.$-ACAGGTACCGCGCGCCTCTTC-3 $\left.3^{\prime}\right)$ and P5864 (5'AGTGGATCCCGGCAAAGTCGCTG) were used to confirm a double crossover event.

\section{Genetic Complementation of the modABC Mutant}

To complement the mod mutant, a $2.6 \mathrm{~kb}$ fragment that contained the putative upstream promoter and coding region of the $\bmod A B C$ genes was amplified from $P$. putida J5 with primers $\mathrm{P} 3210$ and P5864. Primers MJ4-F (5'CT CAAGCTTGGGCAAGCGGCACTCG-3') and MJ4-R $\left(5^{\prime}\right.$-CTCTCTAGAAGCGTGTCGCCATCGC-3') were also used to amplify the $\bmod A B C$ gene from Arthrobacter oxidans J4 to complement the mod mutant. The amplified intact $\bmod A B C$ genes were inserted into the shuttle vector pME6032 or pBBR1MCS2, and the resulting plasmids were introduced into strain $\mathrm{J} 5 \Delta \bmod$ by triparental mating (Wei and Zhang, 2006).

\section{Transcription Analysis of modABC Genes}

For determination of promoter activity, the promoter region that included the upstream fragment of $\bmod A B C$ was amplified by PCR using primers P2925 (5'-GCGTGGGATCCATAATCGGA$\left.3^{\prime}\right)$ and P3505 (5'-CCATTCTGGATCCGCGCATA- $\left.3^{\prime}\right)$, and a 580 bp BamHI fragment was cloned ahead of a promoterless lacZ in p970Km (Yan et al., 2009), which is a derivative plasmid of pRG970 (Van den Eede et al., 1992). The resulting plasmid, p970-PmodABC, was introduced into strain J5 and used to examine the activity of the $\bmod A B C$ promoter. $\beta$-galactosidase measurement was performed at $6 \mathrm{hpi}$ according to the standard protocol.

A further RT-qPCR assay was conducted to check the expression of $\bmod A B C$. Fresh midexponential cells from a single colony of strain J5 were harvested using 12,000 rpm centrifugation. Total RNA was extracted from the pellets using RNAprep pure cell/bacteria kit (Tiangen), treated with DNase, and reverse transcribed to cDNA using random hexamer primers and SuperScript III reverse transcriptase (Invitrogen). Transcriptional expression of $\bmod A$ was determined using the CFX Connect ${ }^{\mathrm{TM}}$ Real-Time System (Bio-Rad) with Luna ${ }^{\circledR}$ Universal qPCR Master Mix (New England Biolabs). Transcript levels for $\operatorname{modA}$ were calculated relative to the level for the housekeeping gene gyrA.

\section{Detection of Nicotine Concentration}

$P$. putida $\mathrm{J} 5$ and its derivatives were cultured to stationary phase in LB medium, and $1 \mathrm{ml}$ of the cell suspension was spun down, inoculated into $100 \mathrm{ml}$ of NI media that contained $1.0 / 1$ nicotine, and incubated at $30^{\circ} \mathrm{C}$. One $\mathrm{mM}$ sodium tungstate and 1,10 , $100 \mu \mathrm{M}$, and $1 \mathrm{mM}$ sodium molybdate were added as needed. To determine cell density, the absorbance $(600 \mathrm{~nm})$ of $3 \mathrm{ml}$ of culture was determined with a spectrophotometer at $2 \mathrm{~h}$ intervals. The cell suspensions were then centrifuged, and the nicotine concentration of the supernatant was determined by high-pressure liquid chromatography (HPLC) (Wei et al., 2009). 


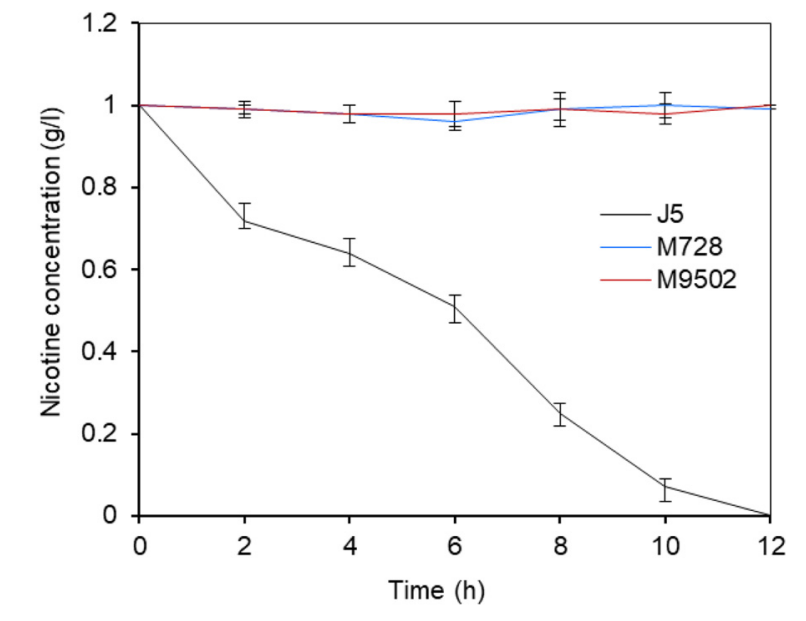

FIGURE 1 | Nicotine degrading efficiency of $P$. putida J5 and the Tn5 insertion mutants M728 and M9502. All strains were grown in $\mathrm{NI}$ medium supplemented with $1 \mathrm{~g} / \mathrm{l}$ nicotine and incubated at $30^{\circ} \mathrm{C}$ and $200 \mathrm{rpm}$. Nicotine degrading efficiency were monitored every $2 \mathrm{~h}$ according to a previous study (Wei et al., 2009). Presented data are average and standard error of the mean for at least three cultures which were assayed in duplicate.

\section{RESULTS}

\section{Isolation and Characterization of Nicotine-Degrading, Deficient Mutants M430, M728, and M9502}

Previous analysis of the mutant strains M430, M728, and M9502 revealed that Tn5 was inserted into the homologs of $\operatorname{modB}$ and modC genes (Xia et al., 2015). Because the Tn5 insertion sites of M430 and M728 were very close to the $\operatorname{modB}$ gene, we only determined the degradation efficiency of mutants M728 and M9502, conducted Southern hybridization analysis, and subsequently cloned a larger fragment that covered the Tn5 transposon.

Under optimal conditions, wild type P. putida strain J5 thoroughly degraded $1.0 \mathrm{~g} / \mathrm{l}$ nicotine in $12 \mathrm{~h}$ (Wei et al., 2009), but the Tn5 mutants, M728 and M9502, completely failed to degrade nicotine (Figure 1). The hybridization patterns of the two mutants were different (Figure 2A), which indicated that the mutants were not identical. Genomic sequences that flanked the insertion sites of M728 and M9502 were cloned by the shot-gun strategy with PstI and EcoRI, based on the results of Southern blotting. The resulting plasmids, pBSM728-P and pBSM9502-E, contained about 6.3 and $3.6 \mathrm{~kb}$ foreign fragments, respectively, which included $1.6 \mathrm{~kb}$ of the mini-Tn5 sequence. After assembling the two fragments and discarding the Tn5 sequence, a $7.0 \mathrm{~kb}$ fragment was obtained (Figure 2B).

\section{modABC Genes}

In the nucleotide sequence of the $7.0 \mathrm{~kb}$ fragment that was "rescued" from M728 and M9502, three open reading frames (ORFs) were found on one strand (Figure 2B). The genomic organization of the three ORFs suggested a transcriptional

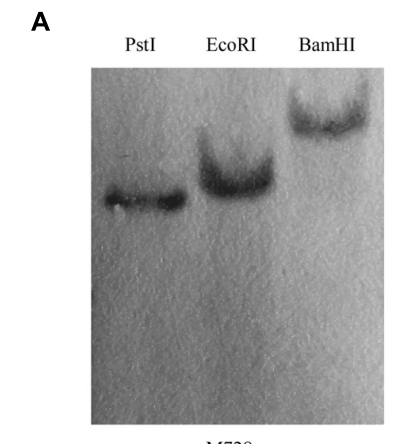

$\mathrm{M} 728$

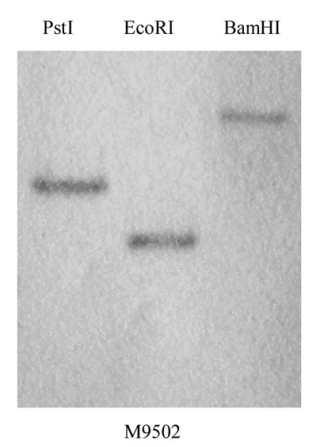

B
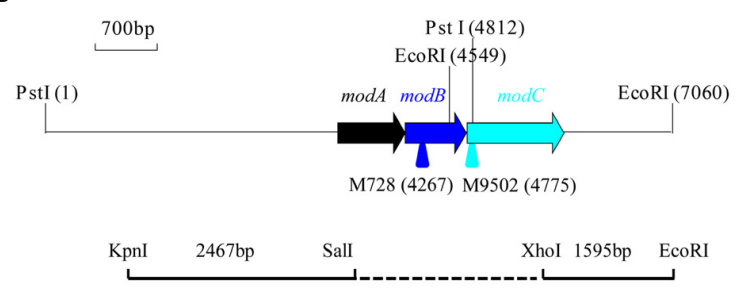

KpnI $4062 \mathrm{bp} \quad$ EcoRI

FIGURE 2 | Southern hybridization of the nicotine-degrading deficient mutants M728 and M9502 and a diagram of the gene cluster of the modABC transport system and construction of the modABC deletion mutant. (A) Only one band was detected from the genomic DNA of M728 and M9502 after digestion with Pstl, EcoRl, and BamHI. (B) Arrows represent the location and orientation of modABC genes. Suicide plasmid pHSG299 that contained the deleted modABC gene was constructed by ligating Kpnl- Sall and XholECORl fragments.

coupling. Potential Shine-Dalgarno sequences were identified at appropriate distances in front of the predicted start codons. These ORFs encoded $252(26.7 \mathrm{kDa}), 229(24.2 \mathrm{kDa})$, and $363(39.4 \mathrm{kDa})$ amino acid residues, which exhibited 88,93 , and $86 \%$ identities with ModA, ModB, and ModC in P. putida S16, respectively, which is a nicotine-degrading bacterium that has had the entire genome sequenced (Yu et al., 2011; Tang et al., 2013). Sequence analysis revealed accurately that the Tn5 cassettes of M728 and M9502 were inserted at the sites of $219 \mathrm{bp}$ of $\operatorname{modB}$ and 36 bp of modC. The deduced $N$-terminus of ModA showed the typical features of lipoproteins (Sutcliffe and Russell, 1995). It resembled the molybdate-specific, periplasmic binding proteins. ModB of strain J5 was similar to the integral-membrane, channelforming proteins of molybdate-specific, ABC transporters. ModC was predicted to be a cytosolic protein with typical consensus sequences for nucleotide binding. Additional studies focused on the function of ModABC in Mo uptake and nicotine biodegradation.

\section{Effect of modABC Mutation on Nicotine-Degrading Activity}

To assess the function of the $\bmod A B C$ genes with respect to the nicotine-degrading activity of $P$. putida J5, the modABC genes were also mutated by in-frame deletions in addition to 
the mod::Tn5 mutation (Figure 2B). The deletion-mutant was confirmed by PCR with primers P3210 and P5864. A $2.6 \mathrm{~kb}$ fragment was amplified from wild type strain J5, but only a $0.8 \mathrm{~kb}$ fragment was amplified from strain J5 $\Delta$ mod, which indicated that a sequence of the $\bmod A B C$ gene that contained approximately $1.8 \mathrm{kp}$ had been deleted from strain J5 as designed.

In contrast to wild type $P$. putida J5, cells of the modABC mutant strain were unable to grow in a minimal medium with nicotine as the sole source of carbon and nitrogen (Figure 3B) but had a similar growth rate to the wild in the medium with glucose (Supplementary Figure S1). After incubating the cells under these conditions, almost no nicotine-degrading activity in cells of the modABC mutant was detected compared with those in cells of strain $P$. putida J5 (Figure 3A). Complementation of $P$. putida J5 $\bmod$ with pME6032 that contained wild-type $\bmod A B C$ genes of strain $\mathrm{J} 5$ restored both nicotine-degrading activity and the ability of the cells to grow in minimal medium with nicotine (Figures 3A,B). Similar results were obtained from complementation of $\triangle \bmod$ with $\bmod A B C$ genes of $A$. oxidans $\mathrm{J} 4$ (Figures 3A,B), which is an efficient nicotine-degrading, grampositive bacterium that was isolated from tobacco rhizospheres. These results indicated that the molybdate transport system was involved in nicotine metabolism in P. putida J5.

\section{Effect of Molybdate on Nicotine Metabolism by $P$. putida J5 and the modABC Mutant}

We tested to determine whether the growth and nicotinedegrading activity of wild type $P$. putida J5 was dependent on molybdate. Therefore, we employed tungstate, the specific antagonist of molybdate (Higgins et al., 1956; Nagel and Andreesen, 1989), to remove the molybdenum trace from the medium. We firstly determined if tungstate had impact on bacterial growth and nicotine metabolism. As expected, no difference was found on growth and metabolism when supplying $1 \mathrm{mM}$ tungstate in LB liquid media with $1 \mathrm{~g} / \mathrm{l}$ nicotine (Supplementary Figure S2). If the nicotine minimal growth medium was supplemented with tungstate (1 $\mathrm{mM})$, no growth or nicotine consumption was found. These results seemed to be specific for nicotine, because no inhibition of growth and nicotine-degrading ability on succinate as a carbon source was detected after adding the same concentration of tungstate (Figure 4). Taken together, the results indicated that molybdate was a key element required by $P$. putida to degrade nicotine.

Although we already proved ModABC constituted a molybdate transport system and played an important role in nicotine biodegradation in P. putida J5, we were not sure if increased concentrations of molybdate complemented the mutation of modABC mutants. Therefore, P. putida J5 $\Delta$ mod was grown in minimal medium supplemented with nicotine and with different concentrations of molybdate. In the presence of molybdate, J5 $\Delta \bmod$ revealed divergent kinetics of nicotine reduction and growth rate (Figure 5). The nicotine-degrading activity of the $P$. putida J5 $\Delta$ mod was not influenced by supplementation with a low concentration of molybdate $(1 \mu \mathrm{M})$.

\section{A}

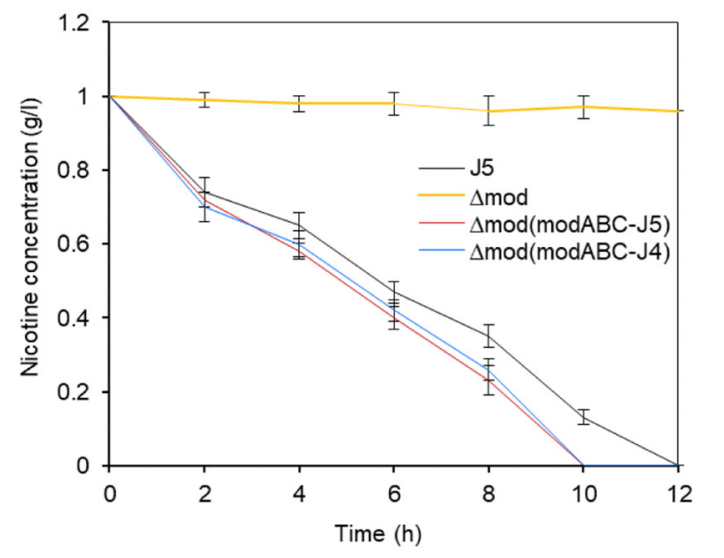

B

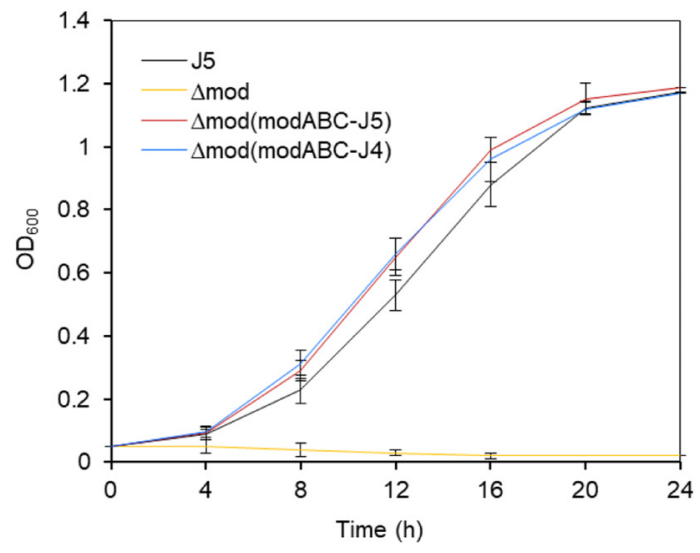

FIGURE 3 | Time course of nicotine biodegradation (A) and growth (B) of P. putida $\mathrm{J} 5$ and the modABC mutants. All strains were grown in $\mathrm{NI}$ medium supplemented with $1 \mathrm{~g} / \mathrm{l}$ nicotine and incubated at $30^{\circ} \mathrm{C}$ and $200 \mathrm{rpm}$. Culture samples were removed periodically to be measured for absorbance at $600 \mathrm{~nm}$ and the residual nicotine with HPLC. Presented data are averages and standard errors of the mean for at least three cultures that were assayed in duplicate.

However, as the molybdate supplementation was increased, $P$. putida $\mathrm{J} 5 \Delta \bmod$ restored nicotine-degrading activity and recovered growth in the nicotine medium. At a concentration of $100 \mu \mathrm{M}$, molybdate had the highest efficiency in supporting the growth and nicotine-degrading activity of the $\bmod A B C$ mutant, while a higher concentration of molybdate up to $1 \mathrm{mM}$ repressed uptake of Mo and resulted in a decreased rate of growth and nicotine-degrading activity. The results clearly indicated that least one other system was able to transport molybdate, but with lower affinity.

\section{Transcription of $\bmod A B C$ Is Molybdate-Dependent}

To investigate whether the expression of $\bmod A B C$ genes was associated with molybdate, the effect of molybdate on the expression of $\bmod A B C$ was first measured using a $\operatorname{Pmod} A B C:$ :lac $Z$ transcriptional fusion in the plasmid p970Km. Before doing this assay, we firstly tested the 


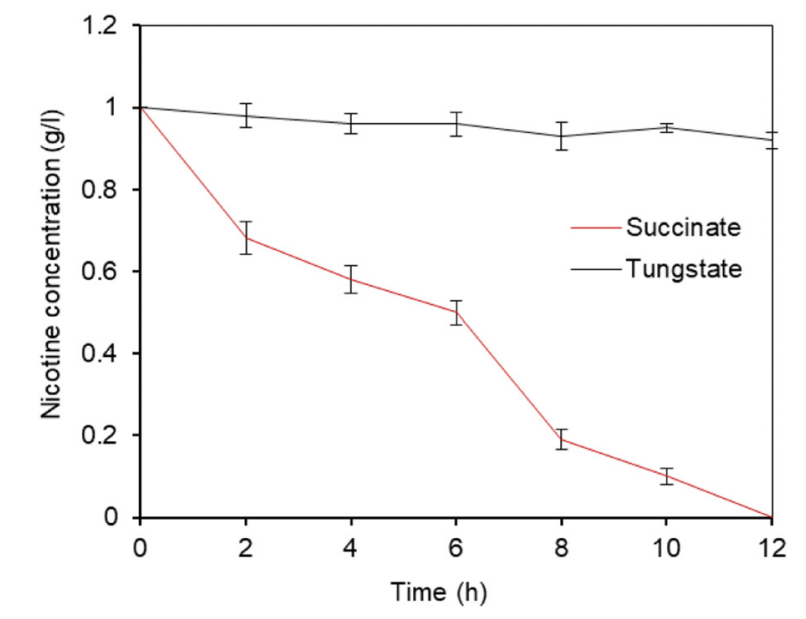

FIGURE 4 | Nicotine degradation of $P$. putida J5 was inhibited by tungstate. $P$. putida $\mathrm{J} 5$ was cultured in $\mathrm{NI}$ medium with or without $1 \mathrm{mM}$ sodium tungstate at $30^{\circ} \mathrm{C}$ and $200 \mathrm{rpm}$. Nicotine-degrading efficiency was monitored every $2 \mathrm{~h}$ as described above. Presented data are averages and standard errors of the mean for at least three cultures that were assayed in duplicate.

growth of the wild type strain J5 supplied with selected titers of molybdate. No significant differences of the growth rates were found between the treatments (data not shown), which dismissed the possibility of growth affecting gene expression. The results revealed that $\bmod A B C$ transcription under low-molybdate conditions was significantly enhanced compared with that under high-molybdate conditions, and $10 \mu \mathrm{M}$ of molybdate induced the highest expression of $\bmod A B C$. After the molybdate concentration was increased to $100 \mu \mathrm{M}$, expression of the $\bmod A B C$ genes were completely repressed (Figure 6A). We also employed RT-qPCR to double-check the expression of $\bmod A$ at different molybdate concentrations. The results were consistent with those in the promoter assay. The only exception was that $\bmod A B C$ was induced moderately at higher molybdate concentrations (Figure 6B), which suggested that qPCR was more sensitive than transcriptional fusion. The expression of $\bmod A B C$ was induced by the depletion of molybdate in P. putida J5, and the modABC molybdate transport system was a high affinity system.

\section{DISCUSSION}

Pseudomonas putida J5 is an efficient nicotine-degrading bacterium, which has been used to degrade employed in nicotine-contained tobacco waste (Wei et al., 2008). The molecular mechanisms of nicotine degradation by $P$. putida J5 was determined extensively by a genome-wide $\operatorname{Tn} 5$ mutagenesis strategy (Xia et al., 2015). In this study, we report on the physiological and genetic analysis of the three Tn5 mutants defective in $\bmod A B C$ that encoded the molybdenum transport system. Both the random and sitedirected mutants failed to degrade nicotine in minimal medium

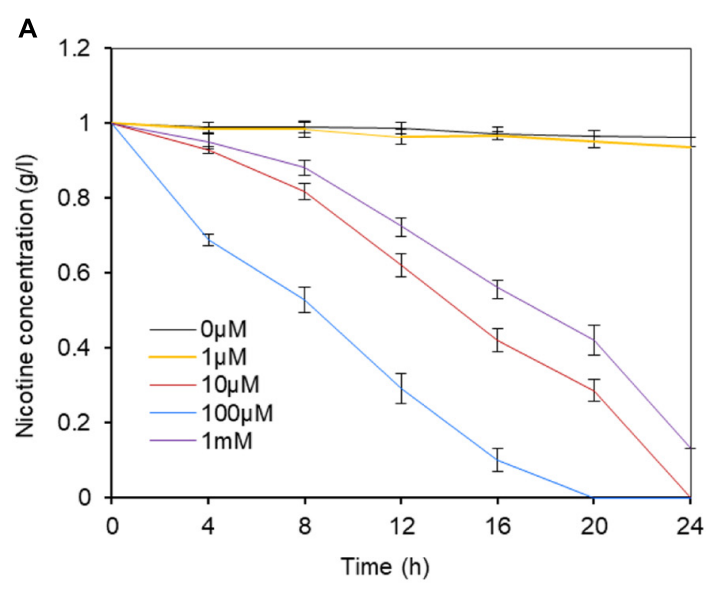

B

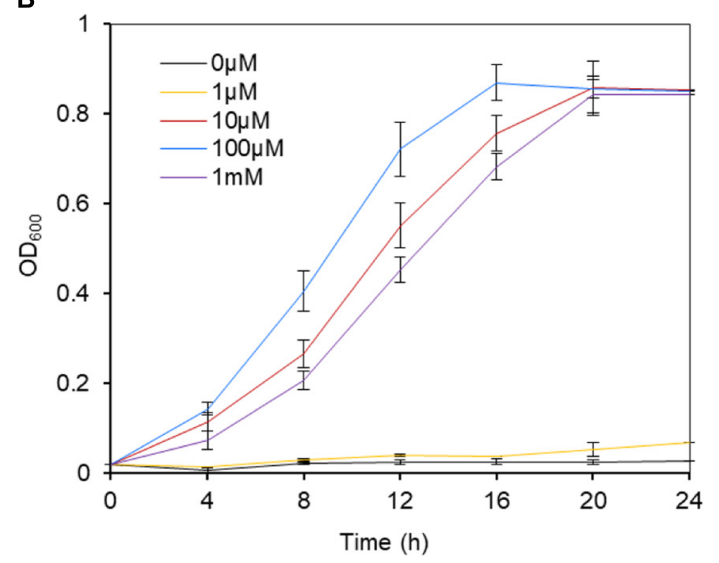

FIGURE 5 | Nicotine degradation (A) and growth (B) of the P. putida J5 modABC mutant at a range of molybdate concentrations. The modABC mutant was cultured in $\mathrm{NI}$ medium supplemented with 1, 10, $100 \mu \mathrm{M}$, and $1 \mathrm{mM}$ sodium molybdate. Culture samples were removed periodically to be measured for absorbance at $600 \mathrm{~nm}$ and the residual nicotine with HPLC. Presented data are averages and standard errors of the mean for at least three cultures that were assayed in duplicate.

with nicotine as the sole source of carbon and nitrogen. Complementation of the mutants with native $\bmod A B C$ genes or the homologs from well-studied $A$. oxidans fully restored nicotine-degrading activity. The effect of molybdate was observed for growth and utilization of the substrate of nicotine, as evidenced by the strong inhibition of tungstate, which was not observed for succinate, a control substrate reported before. The same phenomenon was also reported for degradation of quinolone and 2-furoic acid by Pseudomonas strains (Koenig and Andreesen, 1989; Blaschke et al., 1991). Overall, it seems likely that degradation by $N$-heterocyclic compounds generally involves a molybdenum-dependent reaction.

The trace element molybdenum is an important catalytic component of many enzymes involved in microbial metabolism of nitrogen, sulfur, and carbon. Enzymes that contain Mo at their active sites catalyze oxo-transfer reactions (Zhang et al., 2011). A variety of molybdoenzymes, such as xanthine oxidase, 


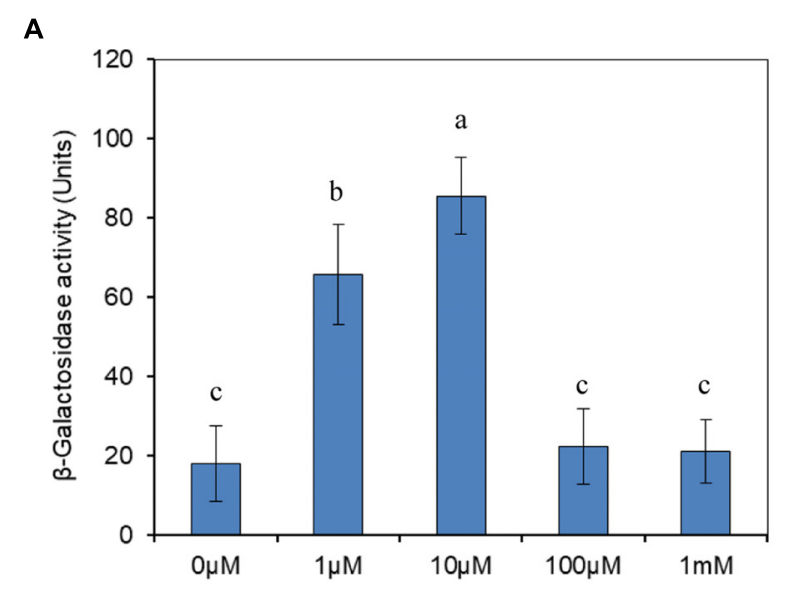

B

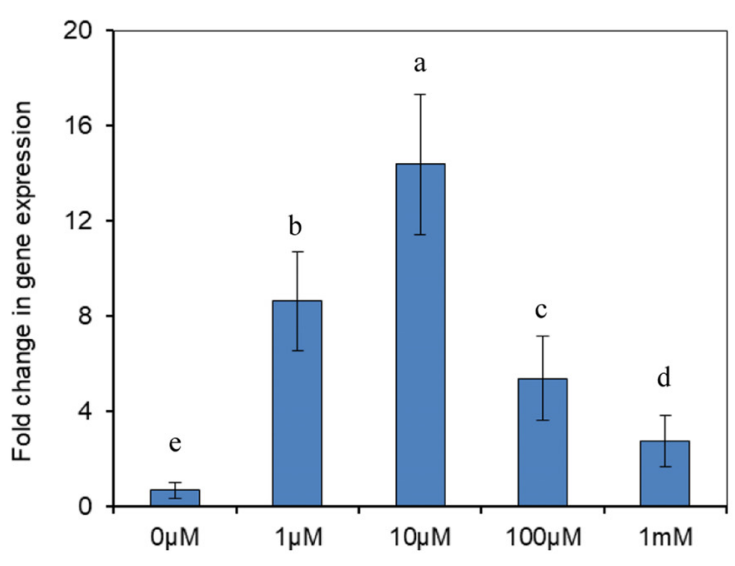

FIGURE 6 | Transcriptional analysis of modABC genes. (A) Expression of $\beta$-galactosidase from a PmodABC::/acZ fusion in $P$. putida J5. Cells were grown aerobically in $\mathrm{NI}$ medium supplemented with different concentrations of molybdate. (B) RT-qPCR analysis of the modA gene transcript produced in

P. putida J5 that was grown as described above. Results presented in these histograms are the means of three independent experiments, and error bars indicate the standard deviations. Different letters represent significant differences between the treatments.

catalyze oxidative hydroxylation of a wide range of aldehydes and aromatic heterocycles (Stephan et al., 1996; Parschat et al., 2003; Wagener et al., 2009; Yokoyama and Leimkuhler, 2015). In the best-elucidated metabolic pathway of nicotine degradation by $A$. nicotinovorans, the heterotrimeric molybdenum enzyme $\mathrm{NDH}$ catalyzed the first nicotine-degrading reaction, which was hydroxylation of the pyridine ring to 6-hydroxynicotine (Brandsch, 2006; Fitzpatrick, 2018). But unfortunately, the nicotine oxidase NdaA from $P$. putida J5 and its homologs Nox from Pseudomonas sp. HZN6 and NicA2 from P. putida S16 have not shown such characters to known molybdoenzymes (Qiu et al., 2013; Tang et al., 2013). However, the key step in nicotine metabolism in the conversion of 3-succinoylpyridine to 6-hydroxy-3-succinoylpyridine by $P$. putida S16 was catalyzed by a multi-enzyme reaction that consisted of a molybdopeterinbinding oxidase (SpmA), molybdopterin dehydrogenase (SpmB), and a (2Fe-2S)-binding ferredoxin (spmC) with molybdenum molybdopterin cytosine dinucleotide as a cofactor (Tang et al., 2013). SpmABC proteins showed significant sequence identities to the subunits of the xanthine dehydrogenase family, such as $\mathrm{NDH}$, a molybdoenzyme of A. nicotinovorans (Brandsch, 2006; Tang et al., 2013). The SpmABC homologs were also found from the draft genome of strain J5, which locates downstream of NadD, a homolog of Sapd in strain S16 (Tang et al., 2013). These reports support our conclusion of molybdenumdependent nicotine degradation by Pseudomonas strains, although the SpmABC products await further purification and characterization.

It is very interesting to find that the ModABC transport system was repressed by a high concentration of molybdate, but $100 \mu \mathrm{M}$ molybdate restored the growth and nicotinedegrading activity of the $\bmod A B C$ mutant. In Staphylococcus carnosus, the mutant of modABC reduced nitrates very slowly in the absence of molybdate and efficiently in the presence of added molybdate (100 $\mu \mathrm{M})$ (Neubauer et al., 1999). In E. coli, the molybdate transport system was repressed by increasing the molybdate concentration in the medium, and the $\bmod A B C$ mutant utilized other transport systems with a lower affinity for molybdate (Rosentel et al., 1995). Molybdate can be taken up by the sulfate-transport system in the absence of a functional, high-affinity, molybdate-transport system in E. coli and B. japonicum (Rosentel et al., 1995; Delgado et al., 2006).

Our results, together with the similarity to previous reports, clearly indicated an involvement of the ModABC of P. putida J5 in molybdate transport. This is the first research to report that molybdenum and the ModABC transport system were involved in nicotine degradation by $P$. putida, but further research needs to be done to elucidate the other nicotine-degrading related ModABC transport systems.

\section{AUTHOR CONTRIBUTIONS}

H-LW and ZX designed the research. ZX, LL, and H-YZ performed the research. H-YZ and H-LW analyzed the data. H-LW wrote the paper.

\section{FUNDING}

This work was supported by the Science and Technology Program of the Yunnan Tobacco (2017YN08, 2017YN10) and the Chinese Academy of Agricultural Sciences Special Support Program for Distinguished Talents (854-3, 866-2).

\section{SUPPLEMENTARY MATERIAL}

The Supplementary Material for this article can be found online at: https://www.frontiersin.org/articles/ 10.3389/fmicb.2018.03030/full\#supplementary-material 
FIGURE S1 | Time course of growth of $P$. putida J5 and the modABC mutants in M9 medium with glucose as the sole source of carbon and nitrogen. All strains were incubated at $30^{\circ} \mathrm{C}$ and $200 \mathrm{rpm}$. Culture samples were removed periodically to be measured for absorbance at $600 \mathrm{~nm}$. Presented data are averages and standard errors of the mean for at least three cultures that were assayed in duplicate.

\section{REFERENCES}

Altschul, S. F., Gish, W., Miller, W., Myers, E. W., and Lipman, D. J. (1990). Basic local alignment search tool. J. Mol. Biol. 215, 403-410. doi: 10.1016/S00222836(05)80360-2

Blaschke, M., Kretzer, A., Schaifer, C., Nagel, M., and Andreesen, J. R. (1991). Molybdenum-dependent degradation of quinolone by Pseudomonas putida Chin IK and other aerobic bacteria. Arch. Microbiol. 155, 164-169. doi: 10.1007/ BF00248612

Brandsch, R. (2006). Microbiology and biochemistry of nicotine degradation. Appl. Microbiol. Biotechnol. 69, 493-498. doi: 10.1007/s00253-005-0226-0

Delgado, M. J., Tresierra-Ayala, A., Talbi, C., and Bedmar, E. J. (2006). Functional characterization of the Bradyrhizobium japonicum modA and modB genes involved in molybdenum transport. Microbiology 152, 199-207. doi: 10.1099/ mic. $0.28347-0$

Figurski, D., and Helinski, D. R. (1979). Replication of an origin-containing derivative of plasmid RK2 dependent on a plasmid function provided in trans. Proc. Natl. Acad. Sci. U.S.A. 76, 1648-1652. doi: 10.1073/pnas.76.4. 1648

Fitzpatrick, P. F. (2018). The enzymes of microbial nicotine metabolism. Beilstein J. Org. Chem. 2018, 2295-2307. doi: 10.3762/bjoc.14.204

Freudenberg, W., König, K., and Andreesen, J. R. (1988). Nicotine dehydrogenase from Arthrobacter oxidans: a molybdenum containing hydroxylase. FEMS Microbiol. Lett. 52, 13-18. doi: 10.1111/j.1574-6968.1988.tb02564.x

Ganas, P., Sachelaru, P., Mihasan, M., Igloi, G. L., and Brandsch, R. (2008). Two closely related pathways of nicotine catabolism in Arthrobacter nicotinovorans and Nocardioides sp. strain JS614. Arch. Microbiol. 189, 511-517. doi: 10.1007/ s00203-007-0340-8

Heeb, S., Itoh, Y., Nishijyo, T., Schnider, U., Keel, C., Wade, J., et al. (2000). Small, stable shuttle vectors based on the minimal pVS1 replicon for use in gramnegative, plant-associated bacteria. Mol. Plant Microbe Interact. 13, 232-237. doi: 10.1094/MPMI.2000.13.2.232

Higgins, E. S., Richert, D. A., and Westerfeld, W. W. (1956). Tungstate antagonism of molybdate in Aspergillus niger. Proc. Soc. Exp. Biol. Med. 92, 509-511. doi: 10.3181/00379727-92-22527

Hille, R. (1996). The mononuclear molybdenum enzymes. Chem. Rev. 96, $2757-$ 2816. doi: $10.1021 / \mathrm{cr} 950061 \mathrm{t}$

Kisker, C., Schindelin, H., and Rees, D. C. (1997). Molybdenum-cofactorcontaining enzymes: structure and mechanism. Ann. Rev. Biochem. 66, 233267. doi: 10.1146/annurev.biochem.66.1.233

Koenig, K., and Andreesen, J. R. (1989). Molybdenum involvement in aerobic degradation of 2-furoic acid by Pseudomonas putida Ful. Appl. Environ. Microbiol. 55, 1829-1834.

Kovach, M. E., Elzer, P. H., Hill, D. S., Robertson, G. T., Farris, M. A., Roop, R. M., et al. (1995). Four new derivatives of the broad-host-range cloning vector pBBR1MCS, carrying different antibiotic-resistance cassettes. Gene 166, 175-176. doi: 10.1016/0378-1119(95)00584-1

Leimkuhler, S., and Iobbi-Nivol, C. (2015). Bacterial molybdoenzymes: old enzymes for new purposes. FEMS Microbiol. Rev. 40, 1-18. doi: 10.1093/femsre/ fuv043

Luque, F., Mitchenall, L. A., Chapman, M., Christine, R., and Pau, R. N. (1993). Characterization of genes involved in molybdenum transport in Azotobacter vinelandii. Mol. Microbiol. 7, 447-459. doi: 10.1111/j.1365-2958.1993.tb0 1136.x

Maupin-Furlow, J. A., Rosentel, J. K., Lee, J. H., Deppenmeier, U., Gunsalus, R. P., and Shanmugam, K. T. (1995). Genetic analysis of the modABCD (molybdate transport) operon of Escherichia coli. J. Bacteriol. 177, 4851-4856. doi: 10.1128/ jb.177.17.4851-4856.1995

Menendez, C., Otto, A., Igloi, G., Nick, P., Brandsch, R., Schubach, B., et al. (1997). Molybdate-uptake genes and molybdopterin-biosynthesis genes on a
FIGURE S2 | Growth curve (up) and nicotine degradation of $P$. putida J5 in LB liquid media plus $1 \mathrm{~g} / \mathrm{l}$ nicotine with or without $1 \mathrm{mM}$ tungstate. All strains were incubated at $30^{\circ} \mathrm{C}$ and $200 \mathrm{rpm}$. Culture samples were removed periodically to be measured for absorbance at $600 \mathrm{~nm}$. Presented data are averages and standard errors of the mean for at least three cultures that were assayed in duplicate.

bacterial plasmid - characterization of MoeA as a filament-forming protein with adenosinetriphosphatase activity. Eur. J. Biochem. 25, 524-531. doi: 10.1111/j. 1432-1033.1997.0524a.x

Nagel, M., and Andreesen, J. R. (1989). Molybdenum-dependent degradation of nicotinic acid by Bacillus sp. DSM 2923. FEMS Microbiol. Lett. 59, 147-151. doi: 10.1111/j.1574-6968.1989.tb03099.x

Neubauer, H., Pantel, I., Lindgren, P. E., and Gotz, F. (1999). Characterization of the molybdate transport system ModABC of Staphylococcus carnosus. Arch. Microbiol. 172, 109-115. doi: 10.1007/s002030050747

Parschat, K., Hauer, B., Kappl, R., Kraft, R., Huttermann, J., and Fetzner, S. (2003). Gene cluster of Arthrobacter ilicis Ru61a involved in the degradation of quinaldine to anthranilate: characterization and functional expression of the quinaldine 4-oxidase qoxLMS genes. J. Biol. Chem. 278, 27483-27494. doi: 10.1074/jbc.M301330200

Qiu, J., Ma, Y., Zhang, J., Wen, Y., and Liu, W. (2013). Cloning of a novel nicotine oxidase gene from Pseudomonas sp. strain HZN6 whose product nonenantioselectively degrades nicotine to pseudooxynicotine. Appl. Environ. Microbiol. 79, 2164-2171. doi: 10.1128/AEM.03824-12

Rosentel, J. K., Healy, F., Maupin-Furlow, J. A., Lee, J. H., and Shanmugam, K. T. (1995). Molybdate and regulation of mod (molybdate transport), fdhF, and hyc (formate hydrogenlyase) operons in Escherichia coli. J. Bacteriol. 177, 4857-4864. doi: 10.1128/jb.177.17.4857-4864.1995

Sambrook, J., and Russell, D. W. (1998). Molecular Cloning: A Laboratory Manual, 3rd Edn. Cold Spring Harbor, NY: Cold Spring Harbor Laboratory Press.

Self, W. T., Grunden, A. M., Hasona, A., and Shanmugam, K. T. (2001). Molybdate transport. Res. Microbiol. 152, 311-321. doi: 10.1016/S0923-2508(01)0 1202-5

Stephan, I., Tshisuaka, B., Fetzner, S., and Lingens, F. (1996). Quinaldine 4oxidase from Arthrobacter sp. Ru61a, a versatile prokaryotic molybdenumcontaining hydroxylase active towards $\mathrm{N}$-containing heterocyclic compounds and aromatic aldehydes. FEBS J. 236, 155-162. doi: 10.1111/j.1432-1033.1996. 00155.x

Sutcliffe, I. C., and Russell, R. R. B. (1995). Lipoproteins of gram-positive bacteria. J. Bacteriol. 177, 1123-1128. doi: 10.1128/jb.177.5.1123-1128.1995

Tang, H., Wang, L., Wang, W., Yu, H., Zhang, K., Yao, Y., et al. (2013). Systematic unraveling of the unsolved pathway of nicotine degradation in Pseudomonas. PLoS Genet. 9:e1003923. doi: 10.1371/journal.pgen.1003923

Van den Eede, G., Deblaere, R., Goethals, K., Van Montagu, M., and Holsters, M. (1992). Broad host range and promoter selection vectors for bacteria that interact with plants. Mol. Plant Microbe Interact. 5, 228-234. doi: 10.1094/ MPMI-5-228

Wagener, N., Pierik, A. J., Ibdah, A., Hille, R., and Dobbek, H. (2009). The MoSe active site of nicotinate dehydrogenase. Proc. Natl. Acad. Sci. U.S.A. 106, 11055-11060. doi: 10.1073/pnas.0902210106

Wang, G., Angermuller, S., and Klipp, W. (1993). Characterization of Rhodobacter capsulatus genes encoding a molybdenum transport system and putative molybdenum-pterin-binding proteins. J. Bacteriol. 175, 3031-3042. doi: 10. 1128/jb.175.10.3031-3042.1993

Wei, H. L., Lei, L., Liu, S., Xia, Z., and Liu, X. Z. (2009). PanB is involved in nicotine metabolism in Pseudomonas putida. Int. Biodeterior. Biodegradation 63, 988-992. doi: 10.1016/j.ibiod.2009.02.010

Wei, H. L., Lei, L., Xia, Z., Liu, S., Liu, P. G., and Liu, X. Z. (2008). Characterization of a novel aerobic nicotine-biodegrading strain of Pseudomonas putida. Ann. Microbiol. 58, 41-45. doi: 10.1007/BF03179443

Wei, H. L., and Zhang, L. Q. (2006). Quorum-sensing system influences root colonization and biological control ability in Pseudomonas fluorescens 2P24. Antonie Van Leeuwenhoek 89, 267-280. doi: 10.1007/s10482-0059028-8

Xia, Z., Zhang, W., Lei, L., Liu, X., and Wei, H. L. (2015). Genome-wide investigation of the genes involved in nicotine metabolism in Pseudomonas 
putida J5 by Tn5 transposon mutagenesis. Appl. Microbiol. Biotechnol. 99, 6503-6514. doi: 10.1007/s00253-015-6529-x

Yan, Q., Gao, W., Wu, X. G., and Zhang, L. Q. (2009). Regulation of the PcoI/PcoR quorum-sensing system in Pseudomonas fluorescens 2P24 by the PhoP/PhoQ two-component system. Microbiology 155, 124-133. doi: 10.1099/ mic.0.020750-0

Yokoyama, K., and Leimkuhler, S. (2015). The role of FeS clusters for molybdenum cofactor biosynthesis and molybdoenzymes in bacteria. Biochim. Biophys. Acta 1853, 1335-1349. doi: 10.1016/j.bbamcr.2014. 09.021

Yu, H., Tang, H. Z., Wang, L. J., Yao, Y. X., Wu, G., and Xu, P. (2011). Complete genome sequence of nicotine-degrading Pseudomonas putida strain S16. J. Bacteriol. 193, 5541-5542. doi: 10.1128/JB.056 63-11
Zhang, Y., Rump, S., and Gladyshev, V. N. (2011). Comparative genomics and evolution of molybdenum utilization. Coord. Chem. Rev. 255, 1206-1217. doi: 10.1016/j.ccr.2011.02.016

Conflict of Interest Statement: The authors declare that the research was conducted in the absence of any commercial or financial relationships that could be construed as a potential conflict of interest.

Copyright $\odot 2018$ Xia, Lei, Zhang and Wei. This is an open-access article distributed under the terms of the Creative Commons Attribution License (CC BY). The use, distribution or reproduction in other forums is permitted, provided the original author(s) and the copyright owner(s) are credited and that the original publication in this journal is cited, in accordance with accepted academic practice. No use, distribution or reproduction is permitted which does not comply with these terms. 